\title{
Automatic tumor detection in ultrasound breast images: a phantom study
}

\begin{abstract}
This study is focused on automatic detection of tumors in Ultrasound breast images in order to help medical doctors in interpretation of such images using Computer-Aided Detection. In this way a set of 6 most popular ultrasound machines were selected and images were captured with sweeping: modes of operation, transducer, frequency and contrast. A multi purpose multi tissue Ultrasound Phantom was used to make a complete set of ultrasound images in BMode. Pre-processing steps such as gamma corrections, contrast stretching and filtering accompanied by morphological Image Processing were among the steps that were applied to find the final image. All output images were reviewed and marked by two experienced radiologists. Statistical analysis showed a sensitivity of $100 \%$ and accuracy of $99 \%$ for proposed work. It also showed that the same procedure can be use for cystic and solid breast masses with small changes.
\end{abstract}

Keyword: Breast cancer; Computer aided detection; Medical image processing; Ultrasound; Ultrasound Phantom 